\title{
Projection of future cancer incidence and new cancer cases in Manitoba, 2006-2025
}

\author{
J. Nowatzki, MSc (1); B. Moller, PhD (2); A. Demers, PhD (1,3)
}

\section{Abstract}

Introduction: Projecting the burden of cancer is important for evaluating prevention strategies and for administrative planning at cancer facilities.

Methods: We projected cancer incidence and counts for the population of Manitoba using population projections from the Manitoba Bureau of Statistics for the years 2006 to 2025 and cancer incidence data from the Manitoba Cancer Registry for the years 1976 to 2005 . Data were analyzed using a version of the age-period-cohort model with recommended modifications that was developed and tested in the Nordic countries.

Results: The overall incidence of cancer in Manitoba is not projected to change substantially from 2006 to 2025 . However, the age-standardized incidence for lung cancer is expected to decrease, particularly for males, highlighting the importance of tobacco prevention. The total number of new cancer cases per year is expected to increase $36 \%$ over the projection period, attributable primarily to demographic changes.

Conclusion: As the population of Manitoba increases, resource and infrastructure planning will need to account for the expected increase in cancer cases.

Keywords: cancer, projections, incidence, burden of disease, Manitoba

\section{Introduction}

Projection estimates of the burden of cancer have significant implications. They are important for administrative planning at cancer facilities, including establishing future policy and research plans. Projections also have scientific implications; by comparing projected numbers to actual cancer outcomes, they can be used as a benchmark against which to evaluate prevention strategies. ${ }^{1}$ In this case, agestandardised rates allow comparison of cancer incidence across populations on a per capita basis.

Projecting cancer rates is challenging because many risk factors have not been identified; for known risk factors, it is difficult to directly measure exposure and impact. Further, changes in prevention and cancer care that may have a significant impact on incidence or mortality are unpredictable. Various statistical methods have been used to project cancer incidence in other countries. ${ }^{2-7}$ The most common cancer projection models use trends in the three time-related variables of age, period and birth cohort as a substitute measurement of the risks associated with cancer incidence and mortality. ${ }^{1,4,5}$ Future rates are predicted by extrapolating from historical trends in the proxy variables of age, period and cohort. Linear changes in cancer incidence across particular age groups, time periods or generations (cohorts) will likely continue for some time, and this is the major assumption made in age-period-cohort modelling. Because only linear changes in the proxy variables can be extended into the future to make predictions, cancer projections are subject to a great deal of uncertainty.

Moller et al. proposed a method for longterm projection of cancer incidence using age-period-cohort modelling after comparing several versions of the model on data from the Nordic countries. ${ }^{8}$ The researchers tested a total of 15 models by comparing predictions based on prior historical data to observed rates, and recommended those models that produced the most accurate predictions. These tested and recommended techniques have been used to project cancer in the Nordic countries ${ }^{4}$ and in England ${ }^{5}$ but have not been used widely in Canada. ${ }^{9}$ Projections for all cancers combined in the Nordic countries (1998-2002 to 2018-2022) suggest that rates have levelled off for males and will level off for females about ten years from the start of the projection period. ${ }^{4}$ Results from projections in England (2004-2020) suggest that incidence of all cancers combined for males may now be starting to decrease after increasing over the past few decades; similarly, incidence of all cancers combined for females are projected to start decreasing in $2015 .^{5}$

The purpose of this paper is to present the results of applying recommended projection methods to the population of Manitoba, Canada, for the period 2006 to 2025. The resulting estimates of the number of new cancer patients in Manitoba over the projection period will help plan for future healthcare needs related to cancer. Further, the results will show how current

Author references:

1. Epidemiology \& Cancer Registry, CancerCare Manitoba, Winnipeg, Manitoba, Canada

2. Clinical and Registry-based Research Department, Cancer Registry of Norway, Oslo, Norway

3. Department of Community Health Sciences, Faculty of Medicine, University of Manitoba, Winnipeg, Manitoba, Canada

Correspondence: Janet Nowatzki, Epidemiology \& Cancer Registry, CancerCare Manitoba, 409 Tache, Room OG008, Winnipeg, MN R2H 2A6;

Tel.: (204) 235-3431; Fax: (204) 231-8188; Email: janet.nowatzki@cancercare.mb.ca 
trends may impact future incidence, and projected rates will serve as a reference point against which to measure future cancer control initiatives in Manitoba.

\section{Methods}

\section{Data}

We obtained cancer incidence counts from the Manitoba Cancer Registry for the years 1976 to 2005. Data were summarized by sex into 5-year periods (1976-1980, ... 2001-2005) and 5-year age groups $(0-4,5-9, \ldots, 80-84$, $85+$ ) and categorized using codes from both the International Statistical Classification of Diseases, Ninth Revision (ICD-9) ${ }^{10}$ and Tenth Revision (ICD-10). ${ }^{11}$ Non-melanoma skin cancers, benign and in situ neoplasms, and those of uncertain behaviour or unspecified nature were excluded from the projections. In order to have enough incident cases to calculate reliable projections, there needed to be at least three age groups to satisfy the condition of having at least 20 cases per period and age group; cancer sites that did not satisfy this requirement were grouped into the category "other." For females, the category "other" included the cancer sites oral cavity and pharynx, esophagus, melanomas of the skin, kidney and renal pelvis, brain and other nervous system, thyroid, Hodgkin's lymphoma and all other and unspecified cancers. For males, the category "other" included the cancer sites esophagus, melanomas of the skin, testis, brain and other nervous system, thyroid, Hodgkin's lymphoma, and all other and unspecified cancers.

Using the Manitoba population projections for 2006 to 2026, ${ }^{12}$ we aggregated projected population data from the main projection scenario for the years 2006 to 2025 and actual population data for the years 1976 to 2005 into 5-year periods and 5-year age groups by sex in the same manner in which we summarized the cancer incidence data.

\section{Modelling of incidence}

We calculated the projected cancer incidence with NORDPRED ${ }^{4}$ in the statistical computing software $\mathrm{R}$ version 2.5.0.* Developed at the Norwegian Cancer Registry, NORDPRED is a package for predicting cancer incidence and mortality. The model used is based on the standard

*www.r-project.org/ age-period-cohort Poisson regression model $^{13}$ and can be written as follows:

$$
R_{a p}=\exp \left(A_{a}+D \cdot p+P_{p}+C_{c}\right)
$$

where $R_{a p}$ is the incidence in age group $a$ and period $p, A_{a}$ is the age component for age group $a, D$ is the common drift parameter accounting for the linear component of the trend in period and cohort (it is not distinguishable as uniquely a period or a cohort effect $\left.{ }^{14}\right), P_{p}$ is the nonlinear period component of period $p$, and $C_{c}$ is the nonlinear cohort component of cohort $c$.

On the recommendation of Moller et al., we modified the model for most cancer sites. ${ }^{8}$ When cancer registry data are available for a period of several decades, it is recommended that cancer projections be calculated with the power model. ${ }^{1}$ To modify the classic age-period-cohort model in this way, we chose a power link function instead of the logarithmic link function. This change serves to dampen any exponential increase in the rates over time. The model is as follows:

$$
R_{a p}=\left(A_{a}+D \cdot p+P_{p}+C_{c}\right)^{5}
$$

This modification cut the drift parameter by $0 \%, 25 \%, 50 \%$, and $75 \%$ in the first, second, third, and fourth 5 -year periods respectively, with the intention of gradually decreasing the effect of current trends, since current trends are unlikely to continue at the same rate.

Finally, if there were significant sharp changes in the historical rates, projections based on the entire set of historical rates would be inaccurate. The test for departure from a linear trend consisted of checking the significance of $S$ in the following model:

$$
R_{a p}=\left(A_{a}+D \cdot p+S \cdot p^{2}+C_{c}\right)^{5}
$$

In cases where $S$ was significant, we used only the trend in the most recent 10 years to project the drift component. This modification was made for the following cancer sites: lung (males), oral cavity and pharynx (males) and breast (females).

The lower age limit was chosen to insure that the number of cases was greater than
20 in all observation periods for each cancer site. Projections for age groups below this limit were based on average rates in the last 10 years.

Models for observed rates for each cancer site were fit by using the greatest prediction base (between four and six 5-year periods) that satisfied a goodness-of-fit test. The parameters of the model for projected rates were derived as described for the Nordic countries as follows: ${ }^{4}$ the age component $A$ was projected directly; the linear drift $D$ was projected with the two modifications described earlier; the nonlinear cohort component $C$ was projected directly for known cohorts and taken to equal the last estimated effect in the model for new cohorts; and the non-linear period component $P$ was taken to equal the last estimated effect in the model for all future periods. Projected incidence was then calculated based on the resulting models.

Spikes in prostate cancer incidence in the late 1980s and the 1990s coincided with the introduction of the prostate-specific antigen (PSA) test, and reflected a screening effect. Using the standard set of assumptions to project prostate cancer would result in projecting a substantial-and unlikelyjump in incidence. With respect to bladder cancer, historical incidence shows a sharp decrease in the last decade because some neoplasms formerly considered invasive bladder cancer were reclassified as in situ neoplasms; in this case, using historical rates would predict a continued sharp decrease that is equally unlikely to occur. Following methods used in Norway and England, ${ }^{5,15}$ we used a constant rate model to project both prostate and bladder cancer. The average rates from the most recent time period (2001-2005) were assumed to remain constant throughout the projection period. Therefore, any projected increases in the number of cancer cases for these sites are due to changes in the population.

We calculated the projected rates for all cancers combined for males and for females by summing the projected rates that were calculated for the individual cancer sites, including "other." Age-standardized incidence rates were calculated using the 1991 Canadian standard population. 
We calculated the projected cancer incidence counts by multiplying the projected rates by the projected population in corresponding future periods. The percent change in cancer incidence counts over the projection period was apportioned into the contribution from change in cancer risk and the contribution from change in demographics (size and age of population). The portion of the change due to change in risk was calculated by subtracting the number of cases that would result from multiplying current incidence by the estimated future population from the estimated number of future cases. Similarly, the portion of the change due to change in population was calculated by subtracting the current number of cases from the number of cases that would result by multiplying the current incidence and the estimated future population. ${ }^{4}$

\section{Results}

Table 1 shows the ICD-10 $10^{+}$codes used to define the cancer sites and the lower limit of the youngest age group included in the projections, the number of 5 -year periods used in the prediction base, and whether or not the recent trend from the previous 10 years or the trend over the entire prediction base was used in the projection.

\section{Cancer incidence}

Overall, the age-standardized incidence in Manitoba for all cancers combined is predicted to decrease slightly over the projection period, 2006 to 2023 (Figure 1). Figure 2 shows the actual (1976-2005) and projected (2006-2025) age-standardized incidence by cancer sites. For most sites, rates are expected to remain stable over the projection period. The largest changes in incidence expected over the 20-year projection period are decreases in oral cavity and pharynx cancers $(-25 \%)$ and lung cancers $(-32 \%)$ among males. Female lung cancer incidence is expected to continue to increase for 5 to 10 years before starting to decrease.

TABLE 1

ICD-10 codes, ${ }^{a}$ lower limit of the youngest age group used in the models, number of periods used in the prediction base, and use of recent or average trend in cancer projections, Manitoba

\begin{tabular}{|c|c|c|c|c|c|c|c|}
\hline Cancer site & ICD-10 codes $^{\mathrm{a}}$ & 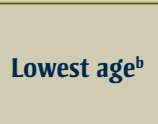 & $\begin{array}{c}\text { Number of } \\
\text { periods }{ }^{c}\end{array}$ & $\begin{array}{l}\text { Average trend } \\
\text { or recent } \\
\text { trend }^{\mathrm{d}}\end{array}$ & 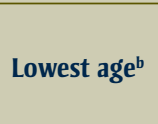 & $\begin{array}{c}\text { Number of } \\
\text { periods }^{c}\end{array}$ & $\begin{array}{l}\text { Average trend } \\
\text { or recent } \\
\text { trend }^{\mathrm{e}}\end{array}$ \\
\hline Oral cavity and pharynx & $\mathrm{C} 000-\mathrm{C} 148$ & - & - & - & 45 & 6 & Recent \\
\hline Stomach & $\mathrm{C} 16$ & 70 & 6 & Average & 55 & 6 & Average \\
\hline Pancreas & $\mathrm{C} 25$ & 65 & 6 & Average & 60 & 6 & Average \\
\hline Lung & C34 & 45 & 6 & Average & 45 & 6 & Recent \\
\hline Breast & C50 & 30 & 4 & Recent & - & - & - \\
\hline Cervix uteri & C53 & 30 & 6 & Average & - & - & - \\
\hline Urinary bladder & C66-C68 & - & - & - & - & - & - \\
\hline Kidney and renal pelvis & C64, C65 & - & - & - & 50 & 6 & Average \\
\hline Non-Hodgkin's lymphoma & C82-C85, C96.3 & 55 & 6 & Average & 60 & 6 & Average \\
\hline Leukemia & C90.1, C91-C95 & 65 & 6 & Average & 55 & 6 & Average \\
\hline Other $^{f}$ & & 15 & 6 & Average & 15 & 6 & Average \\
\hline
\end{tabular}

Abbreviations: ICD-10, International Statistical Classification of Diseases and Related Health Problems, 10th Revision; ICD 9, International Statistical Classification of Diseases and Related Health Problems, 9th Revision.

a The more recent ICD-10 have been in use since 2002. ICD-10 codes map over easily to ICD-9, and hence only they are shown in Table 1 .

${ }^{\mathrm{b}}$ Lower limit of youngest age group used in the model.

c Number of periods used in the prediction base.

${ }^{\mathrm{d}}$ Average trend over entire prediction base.

${ }^{\mathrm{e}}$ Average trend from last 10 years used in the model.

${ }^{\mathrm{f}}$ Females: C000-C148, C15, C44, C64, C65, C70-73, C81, and all other invasive cancers, not listed here or above (ICD-10: C00-C97). Males: C15, C44, C62, C70-73, C81, and all other invasive cancers, not listed here or above (ICD-10: C00-C97). Our analyses excluded non-melanoma skin cancers.

${ }^{\dagger}$ The more recent ICD-10 codes, in use since 2002, map over easily to the previous ICD-9 codes; hence only they are shown in Table 1. 


\section{Cancer incidence and attributing change}

Table 2 summarizes the predicted change in number of cancer cases by site and gender between the midpoint of the last 5-year period of data (2003) and the midpoint of the last 5-year period of the projections (2023). For each cancer site, it shows the percent of the total change that is attributable to change in risk and the percent of the total change that is attributable to change in the size and age of the population. We expect notable decreases in risk of incidence for the following cancers: oral cavity and pharynx (males), stomach (females), colorectal (females), pancreas (males and females), and lung (males). We also expect notable increases in risk for NonHodgkin's lymphoma in both males and females. The predicted increases in cancer cases for most cancer sites are largely attributable to demographic changes; the population of Manitoba is expected to grow approximately 22\% (1 178460 in 2006 to 1439150 in 2025), largely due to international immigration that is expected to account for more than $80 \%$ of the growth in the population over the projection period. ${ }^{12}$ Simultaneously, this population is expected to age so that the median age increases by 1.3 years over the projection period. ${ }^{12}$ Figure 3 shows the expected

FIGURE 1

Actual and projected age-standardised incidence of all cancers combined until 2025, Manitoba

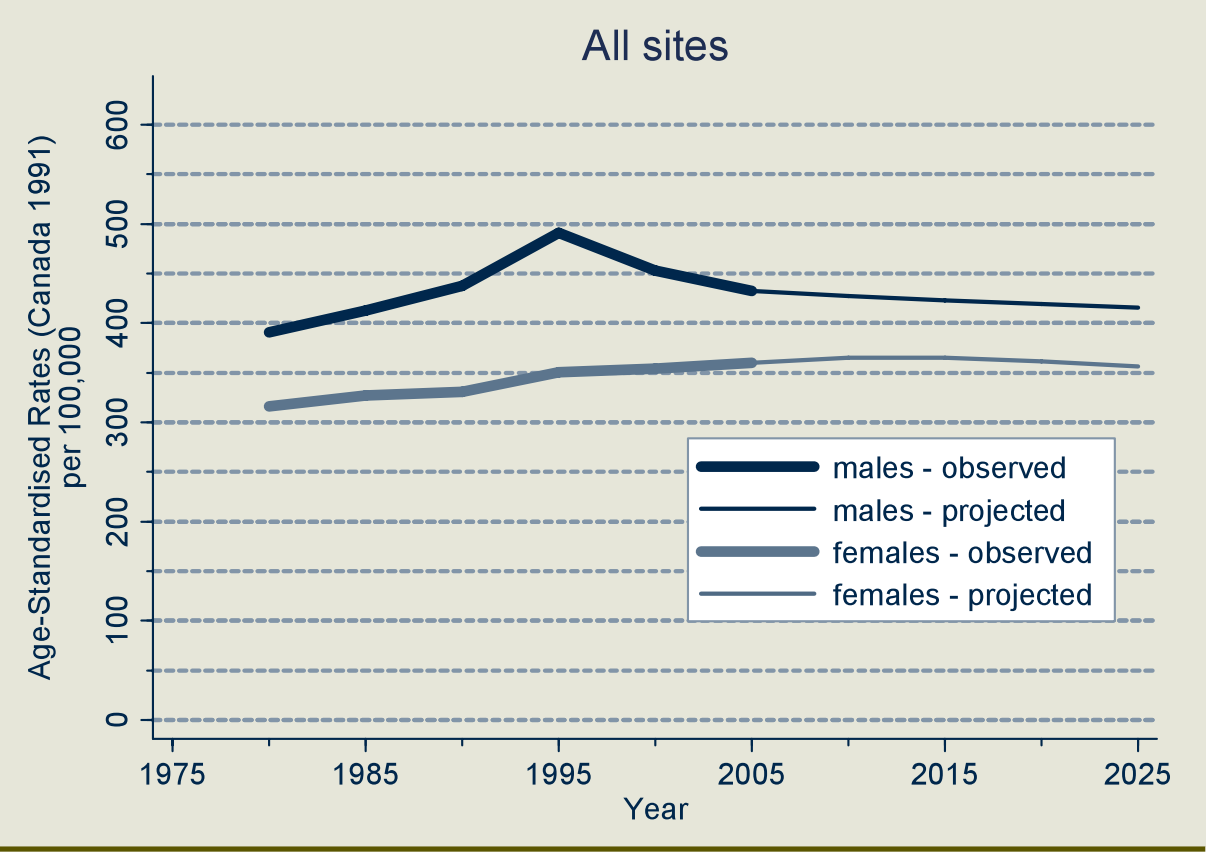

changes in the population of Manitoba by age and gender.

Overall, the total number of new cancer cases per year is expected to increase by $36 \%$ from approximately 5500 in 2003 to approximately 7500 in 2023. Although the numbers of cancer cases in men and women have been relatively similar in recent years, we expect a greater increase in cancer cases in men $(40 \%)$ than in women (30\%) over the projection period, so that by 2023 there will be $7 \%$ more cancer cases in men than in women.

\section{Discussion}

In Manitoba, the incidence of all cancers combined is expected to decrease slightly between 2006 and 2025, similar to the results for England where overall incidence is projected to stabilize and start to decline immediately for men and from 2015 for women. ${ }^{5}$ The expected rates for all cancers combined and for the individual sites will provide a benchmark against which to measure the impact of prevention strategies in Manitoba. For example, targeting risk factors such as obesity, physical inactivity and tobacco use may result in incidence lower than that predicted. in Manitoba to increase from approximately 5500 to 7500 over the projection period. For most cancers, the majority of this projected change is attributable to the changes in the population. As the population of Manitoba increases and slowly ages, cancer services will need to expand to accommodate the predicted increase in cancer cases. The relative contribution to the total number of cancer cases by each of the four major sites (colorectal, lung, breast and prostate) is not expected to change significantly over the projection period. This information is useful for planning the allocation of future cancer services in Manitoba.

The method used to calculate the current predictions has been shown to be fairly accurate. ${ }^{8}$ The discrepancies between actual outcomes and projected numbers in the analysis of the rates in Nordic countries were generally between $10 \%$ and $20 \%$. However, the projected rates and counts for all cancers combined are likely more 
FIGURE 2

Actual and projected age-standardised incidence of most cancer sites to 2025, Manitoba
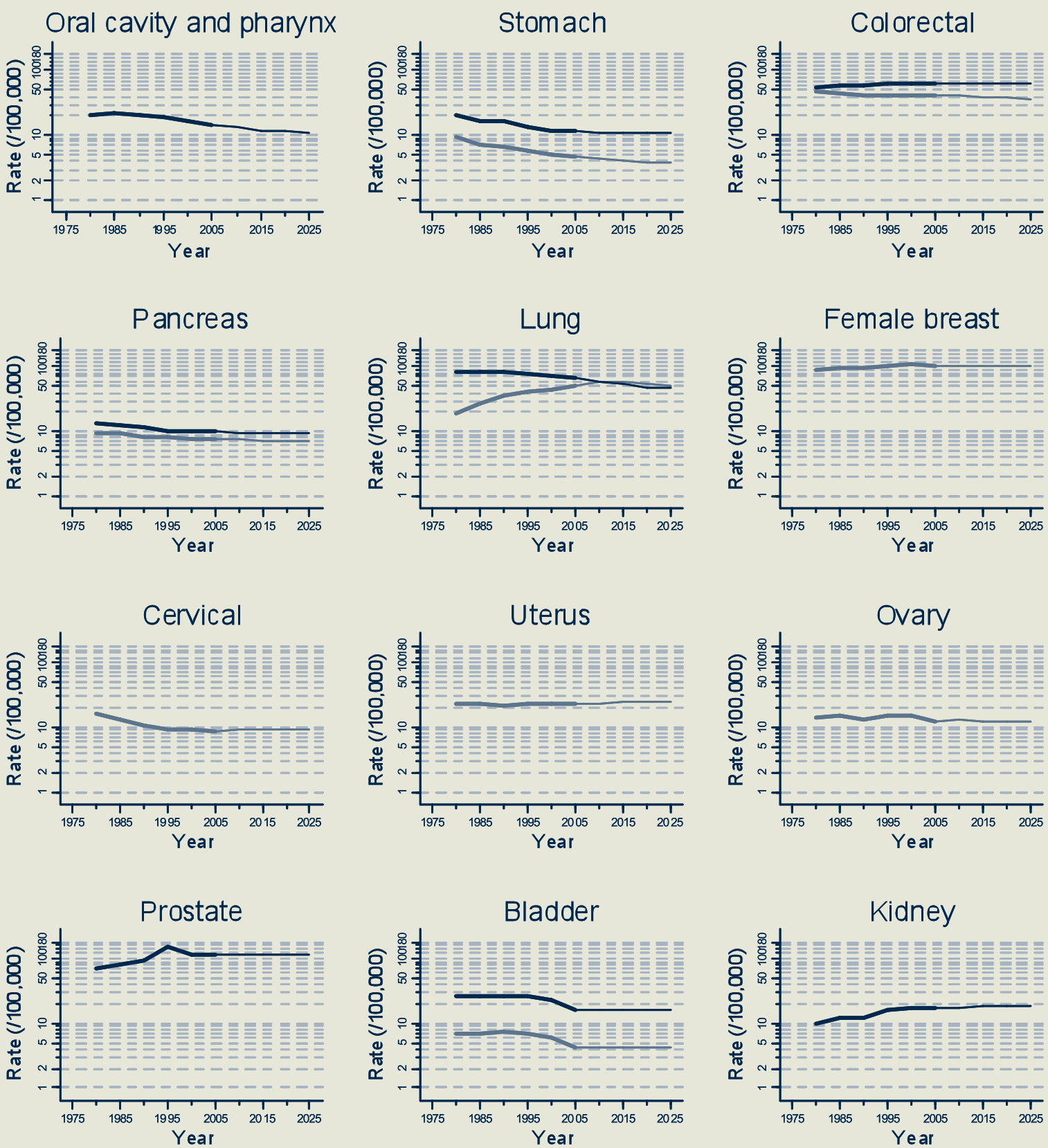

Non-Hodgkin's Iymphoma
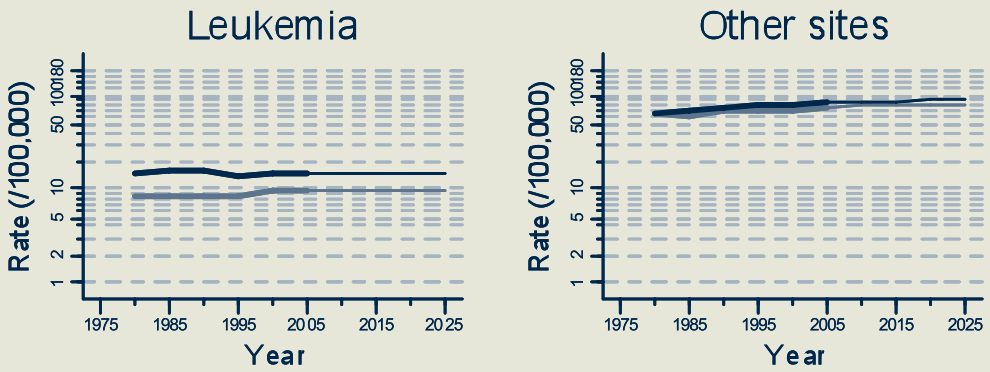

Notes: Male rates in dark blue; female rates in grey

Thick lines represent actual rates; thin lines represent projected rates.

Other sites means all cancers excluding non-melanoma skin cancers, benign and in situ neoplasms, and those of uncertain behaviour or unspecified nature. 
TABLE 2

Number of predicted cancer cases by site and gender in 2023, Manitoba

\begin{tabular}{|c|c|c|c|c|c|c|c|c|c|c|}
\hline \multirow{3}{*}{ Cancer site } & \multicolumn{5}{|c|}{ Females } & \multicolumn{5}{|c|}{ Males } \\
\hline & \multicolumn{2}{|c|}{ Number of cases } & \multicolumn{3}{|c|}{ Percent change (\%) } & \multicolumn{2}{|c|}{ Number of cases } & \multicolumn{3}{|c|}{ Percent change (\%) } \\
\hline & $2003^{a}$ & $2023^{b}$ & Overallc & $\begin{array}{l}\text { Due to } \\
\text { risk }^{\mathbf{d}}\end{array}$ & $\begin{array}{l}\text { Due to change } \\
\text { in population }\end{array}$ & $2003^{a}$ & $2023^{b}$ & Overallc & $\begin{array}{c}\text { Due to } \\
\text { risk }^{\mathrm{d}}\end{array}$ & $\begin{array}{l}\text { Due to change } \\
\text { in populatione }\end{array}$ \\
\hline Oral Cavity and Pharynx & - & - & - & - & - & 97 & 101 & 3 & -37 & 40 \\
\hline Stomach & 42 & 41 & -1 & -28 & 27 & 77 & 106 & 38 & -6 & 44 \\
\hline Colorectal & 358 & 429 & 20 & -11 & 31 & 409 & 622 & 52 & 7 & 45 \\
\hline Pancreas & 69 & 79 & 15 & -16 & 31 & 65 & 88 & 36 & -10 & 46 \\
\hline Lung & 408 & 568 & 40 & 1 & 39 & 434 & 441 & 2 & -47 & 49 \\
\hline Breast & 776 & 982 & 27 & -4 & 31 & - & - & - & - & - \\
\hline Cervix uteri & 53 & 67 & 28 & 4 & 24 & - & - & - & - & - \\
\hline Corpus and uterus & 175 & 248 & 42 & 7 & 35 & - & - & - & - & - \\
\hline Ovary & 94 & 117 & 24 & -7 & 31 & - & - & - & - & - \\
\hline Prostate & - & - & - & - & - & 703 & 1078 & 53 & 0 & 53 \\
\hline Urinary bladder & 37 & 49 & 33 & 0 & 33 & 106 & 152 & 43 & 0 & 43 \\
\hline Kidney and renal pelvis & - & - & - & - & - & 113 & 169 & 49 & 6 & 43 \\
\hline Non-Hodgkin's lymphoma & 119 & 174 & 45 & 16 & 29 & 132 & 204 & 54 & 13 & 41 \\
\hline Leukemia & 74 & 97 & 31 & 2 & 29 & 96 & 134 & 40 & -2 & 42 \\
\hline Other & 574 & 775 & 35 & 6 & 29 & 537 & 782 & 46 & 6 & 40 \\
\hline All cancers ${ }^{f}$ & 2779 & 3625 & 30 & -1 & 31 & 2769 & 3877 & 40 & -6 & 46 \\
\hline
\end{tabular}

a Average annual incidence as recorded 2001-2005.

${ }^{\mathrm{b}}$ Average annual incidence as predicted 2021-2025.

c Overall percent change in the number of cases projected for 2021-2025 compared to the actual number of cases 2001-2005.

${ }^{d}$ Percent of the total change in the number of cases projected due to changes in risk.

${ }^{\text {e }}$ Percent of the total change in the number of cases projected due to changes in the size and age of the population.

${ }^{\mathrm{f}}$ All cancers combined, excluding non-melanoma skin cancers, benign and in situ neoplasms, and those of uncertain behaviour or unspecified nature; totals are a combination of counts and proportions from individual cancer sites.

accurate than those for individual sites, as the variation from individual sites will likely cancel out or stabilize when sites are combined into one category.

The constant rate model was chosen to predict prostate and bladder cancer incidence to better control for short temporal variations in rates breaking away from the general trend. For prostate cancer, it is important to acknowledge that future trends are subject to uncertainty and may depend in part on future use of the PSA test.

\section{Limitations}

Although the method developed in the Nordic countries is recommended for cancer projections, it is not without limitations.
First, the projected rates are based on the assumption that past trends will continue into the future. Any changes in these trends will mean that the projections will not be realized. This is always the case when attempting to predict future events involving uncertainty. Further, projections are likely to be more accurate in the short-term than in later time periods, as trends are more likely to change over longer periods of time. The decreasing reliability of cancer predictions over time is also related to the decreasing reliability of the population predictions. Specifically, changes to the population are cumulative, causing a decrease in the reliability of the predicted numbers over time.
Another limitation in the projections comes from the small size of Manitoba's population. In particular, projections were limited to those sites with enough cases to satisfy the requirements of the prediction model (a minimum of three age groups with at least 20 cases per calendar period and age group). All other sites were combined into one category called "Other," and hence individual trends for these sites were not projected. Despite this, for those cancer sites with sufficient numbers, the patterns in the projected age-standardized rates were generally consistent with other published predictions. ${ }^{5}$ 
FIGURE 3

Actual and projected population of Manitoba by age and sex: $2003^{\mathrm{a}}$ and $2023^{\mathrm{b}}$

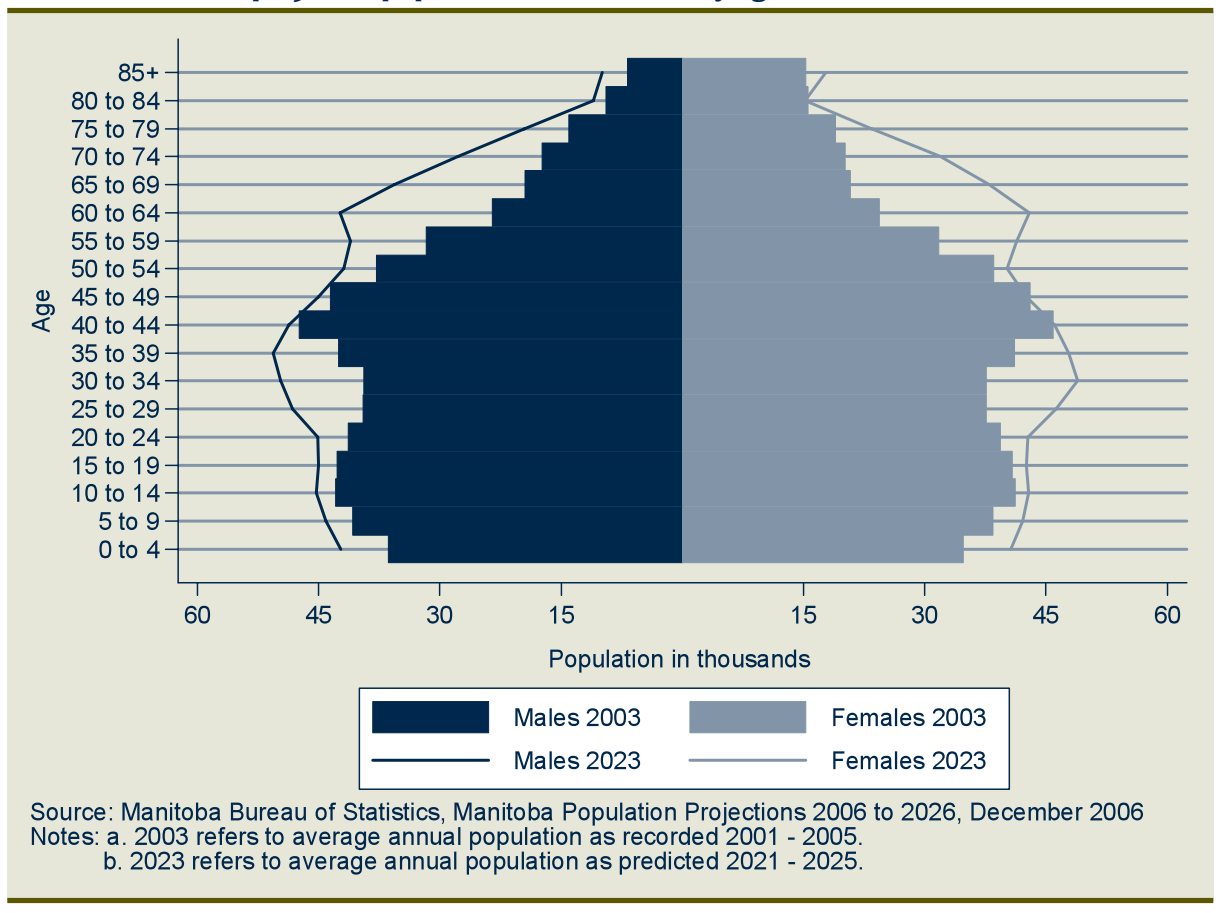

\section{Conclusion}

This study demonstrates that cancer projections can be used as a benchmark against which to estimate the impact of cancer prevention initiatives. Total agestandardized incidence for cancer in Manitoba is expected to decrease slightly over the next 20 years, with reduction in tobacco use likely being the largest attributable factor. The introduction of new and effective prevention measures may be necessary to increase this downward trend. This study also provides useful information for planning for future provision of cancer services in Manitoba based on the expected increase in the number of people diagnosed with cancer as the population of the province grows.

\section{References}

1. Bray F, Moller B. Predicting the future burden of cancer. Nat Rev Cancer. 2006;6:63-74.

2. Clements MS, Armstrong BK, Moolgavkar SH. Lung cancer rate predictions using generalized additive models. Biostatistics. 2005;6:576-89.
8. Moller B, Fekjaer H, Hakulinen T, Sigvaldason H, Storm HH, Talback M, Haldorsen T. Prediction of cancer incidence in the Nordic countries: empirical comparison of different approaches. Stat Med. 2003;22(17):2751-66.

9. Stewart P, Xie L. Results of survey on cancer projection methods used in the provincial/territorial cancer registries. Presented at: Canadian Cancer Statistics Steering Committee Meeting; June 23, 2009; Toronto, ON.

10. World Health Organization. Manual of the international statistical classification of diseases, injuries and causes of death. $9^{\text {th }}$ rev. Geneva: World Health Organization; 1977.

11. World Health Organization. International classification of diseases [Internet]. Geneva: WHO; 2010 [cited 2010 Feb 10]. Available from: http://www.who.int/classifications/ icd/en/

12. Manitoba Bureau of Statistics. Manitoba Population Projections 2006 to 2026. Winnipeg (MB): Manitoba Bureau of Statistics; 2006 Dec. of skin cancer incidence in the Netherlands up to 2015. Br J Dermatol. 2005;152:481-8.

4. Moller B, Fekjaer H, Hakulinen T, Tryggvadottir L, Storm HH, Talback M, Haldorsen T. Prediction of cancer incidence in the Nordic countries up to the year 2020. Eur J Cancer Prev. 2002;11 Suppl 1:S1-S96.

5. Moller H, Fairley L, Coupland V, Okello C, Green M, Forman D, Moller B, Bray F. The future burden of cancer in England: incidence and numbers of new patients in 2020. Br J Cancer. 2007;96:1484-8.

6. New Zealand Ministry of Health. Cancer in New Zealand: trends and projections [Internet]. Ministry of Health (NZ); 2002 Nov [cited 2010 Jun 9]. Available from: http://www.moh.govt.nz/moh.nsf/ 0/8e1d731682cab3d9cc256c7e00764a23?

7. Smith BD, Smith GL, Hurria A, Hortobagyi GN, Buchholz TA. Future of cancer incidence in the United States: burdens upon an aging, changing nation. J Clin Oncol. 2009;27:2758-65.
13. Osmond C. Using age, period and cohort models to estimate future mortality rates. Int J Epidemiol. 1985;14(1):124-9.

14. Clayton D, Schifflers E. Models for temporal variation in cancer rates. II: Age-period-cohort models. Stat Med. 1987;6(4):469-81.

15. Moller B, Aagnes B. Predictions of cancer incidence by health region 2010-2020. In: Cancer in Norway 2005: cancer incidence, mortality, survival and prevalence in Norway [Internet]. Oslo: Cancer Registry of Norway, 2006 [cited 2010 Jun 9]. Available from: http://www.kreftregisteret.no/en/ General/Publications/Cancer-in-Norway/ Cancer-in-Norway-2005/

16. H. Krueger \& Associates Inc. An overview of selected cancers and the modifiable cancer risk factors in Canada. Toronto (ON): The Primary Prevention Action Group of the Canadian Partnership Against Cancer; 2008 May. 
17. H. Krueger \& Associates Inc. Making the Case for Primary Prevention: An Economic Analysis of Risk Factors in Manitoba. Winnipeg (MB): The Heart and Stroke Foundation of Manitoba. 2010 Sept.

18. Rehm J, Baliunas D, Brochu S, Fischer B, Gnam W, Patra J, Popova S, SarnocinskaHart A, Taylor B, Adlaf E, Recel M, Single E. The costs of substance abuse in Canada 2002 [Internet]. Ottawa (ON): Canadian Centre on Substance Abuse. 2006 Mar [cited 2010 Feb 2]. Available from: http://www.ccsa.ca/ Eng/Priorities/Research/CostStudy/Pages/ default.aspx 